\title{
GUEST EDITORIAL \\ Special Section: Topological representation and reasoning in design and manufacturing
}

\author{
DAN BRAHA* \\ Department of Industrial Engineering, Ben-Gurion University, Beer-Sheva 84105, Israel
}

Topology deals with geometric properties that are dependent only on the relative positions of the components of figures and not on such concepts as length, size, and magnitude. Topology supports design and representation of mechanical devices, communication and transportation networks, topographic maps, and planning and controlling of complex activities. In addition, aspects of topology are closely related to symbolic logic, which forms the foundation of artificial intelligence. By approaching engineering design from this abstract point of view, it is possible to use

*Also Affiliate of the New England Complex Systems Institute, $24 \mathrm{Mt}$. Auburn St., Cambridge, Massachusetts, 02135, U.S.A.

Reprint requests to: Dan Braha, Department of Industrial Engineering, Ben-Gurion University, P.O.B. 653, Beer-Sheva 84105, Israel. E-mail: Brahad@bgumail.bgu.ac.il topological methods to study collections of geometric objects or collections of entities that are of concern in design analysis or synthesis. The importance of topological representation and reasoning in analysis, design, and manufacturing is heightened by the contemporary view that stresses the need for conceptual design.

The first special issue on "Topological Representation and Reasoning in Design and Manufacturing" appeared in the 2000 issue, number 5. The second special issue is included herein. The third special issue will appear in the 2001 issue, number 2 of AIEDAM. This series of special issues is oriented toward the exploration of recent advances in Artificial Intelligence related to Topological Design and Manufacturing, and we hope that it will stimulate further research in this area as a unifying design abstraction. 